\title{
Use of geo-information systems in the registration of road-traffic accidents on the roads
}

\author{
Sirojiddin Yadgarov", Qurbon Muminov, Qaxramon Ergashev, and Ruzigul Sayfutdinova
}

Tashkent State Transport University, Tashkent, Uzbekistan

\begin{abstract}
We come up with the use of traditional methods in recording road traffic accidents that occur on highways, which makes the YTH analysis several difficulties and shortcomings. The analysis of YTH s has been shown to be effective in the use of Geoinformation systems. In conclusion, it can be noted that international experience shows that in recent years in European countries with well-developed motorization with high GDP and the level density of the road network, a national policy, and programs in the field of road safety in the medium and long term, in addition to the General problems and methods of their solutions, identifies targets, characterizing the level of road safety. This must include experience in implementing similar programs in other countries with a similar rate of car ownership, income level of the population, the condition, and development of the road network. The level of development of the road network, which is characterized by compliance with the total length, density, distribution of roads by functional value and categories, socioeconomic needs of society in road transport, plays a significant role in the formation of accidents.
\end{abstract}

\section{Introduction}

More than 2,200 people die in road accidents every year, and more than 12,000 people are injured on the republic's roads. In Russia, losses from road accidents account for 4.5 percent of gross domestic product (GDP), while in Sweden, they account for 1 percent of GDP. The number of deaths per 1 car in Russia is 3-5 times higher than the average value of this figure for EU member states [1,2].

Road safety is also a public health issue. Recent research on the global significance of injuries by Harvard University in the United States on behalf of the World Health Organization and the World Bank has shown that road deaths are the leading cause of death among adults aged 15-44 years. Moreover, the same research has shown that by 2020 . Traffic accidents are the third leading cause of death worldwide after heart disease and depression $[3,5]$.

In conclusion, it can be noted that international experience shows that in recent years in European countries with well-developed motorization with high GDP and the level density

\footnotetext{
* Corresponding author: sirojtdtutf@mail.ru
} 
of the road network, a national policy, and programs in the field of road safety in the medium and long term, in addition to the General problems and methods of their solutions, identifies targets, characterizing the level of road safety. This must include experience in implementing similar programs in other countries with a similar rate of car ownership, income level of the population, the condition, and development of the road network [8].

The number of cars around the world is growing day by day. This is primarily the case in the Republic of Uzbekistan. After gaining independence, the automotive industry was developed to strengthen the country's economy.

\section{Materials and Methods}

It is possible to point out three specific features of car traffic that lead to low levels of traffic safety.

1) Insufficient provision of road transport with high-performance road-performance indicators.

2) Insufficient separation of motor traffic from other road users and low traffic culture in Riyadh.

3) The popularity of the driving profession and many low-skilled and low-skilled amateur drivers among them.

Accidents on highways, city streets, and squares resulting in death, injury, damage to vehicles and their cargo, damage to artificial structures, or other material damage resulting from the violation of the normal mode of movement of vehicles (traffic accident). According to this definition, a traffic accident is characterized by the presence of two factors: death of a person, bodily injury or amount of material damage, as well as the presence of any vehicle in motion.

It is observed that one or more elements of A-H-Y-P-M, which are part of the general system, together with the formation of traffic accidents, cause a violation of the normal mode of movement.

Each traffic accident can be conditionally divided into three phases: initial, culmination, and final. They are interdependent, and one can be a continuation of the other.

The initial phase of a traffic accident is the movement of cars and pedestrians on the eve of a dangerous situation.

In a catastrophic situation, the technical capacity of the participants in the action to obtain the name of the traffic accident will not be sufficient, and it will happen.

The culmination phase of traffic accidents is characterized by the occurrence of serious consequences (vehicle breakdown. injury, or death of pedestrians and drivers). This phase can last for several minutes and in bad weather for up to a few minutes. In this case, mainly several vehicles are involved, and it is often called "chain" traffic.

The final phase of the traffic accident coincides with the end of the vehicle stop after the culmination phase. At some point, the final phase continues even if the vehicle stops moving - for example, fires in an overturned car.

A major role in ensuring traffic safety is also played by the highway's main technical and operational indicators. These include the geometric dimensions of the roadbed, the width, and condition of the roadsides, the evenness, and roughness of the pavement, visibility on curves in the plan and longitudinal profile, the illumination of road sections at night, the presence of markings on the roadway, the quality of engineering equipment, the availability of controls following the actual traffic intensity. The influence of road conditions on traffic safety is laid down in the process of road design and is implemented in the process of road operation [6].

The level of development of the road network, which is characterized by compliance with the total length, density, distribution of roads by functional value and categories, 
socio-economic needs of society in road transport, plays a significant role in the formation of accidents. The disproportions in the development of the road network directly affect the unevenness of traffic loading and stimulate the increase in the risk of accidents, especially in areas where the capacity does not meet the observed traffic intensity [7].

According to the current regulations, there are 9 types of traffic:

1. Collision. This includes a collision of vehicles while moving on the opposite side, in one direction, or from the side, and a collision of a motor vehicle with a rail vehicle. This type of traffic accident also includes a collision with a vehicle that suddenly stops.

2. Overturning. It was overturning a moving vehicle, losing its stability. This type of road does not include a collision, overturning of a vehicle due to a collision with a parked vehicle, or an obstacle. Overturning is mainly involved in a single vehicle.

3. Hitting a parked vehicle. The collision of a moving vehicle with a stationary $(\mathrm{V}=0)$ vehicle. This type of accident does not include a sudden (sudden) collision of a vehicle.

4. Hitting obstacles. Vehicles are hitting immovable obstacles (bridge piers, poles and mast supports, road barriers, trees, etc.).

5. Crushing (hitting) pedestrians. This includes hitting a pedestrian or hitting a pedestrian with a vehicle and injuring a pedestrian from the load being transported (timber, pipe, slab, etc.).

6. Pressing (hitting) a cyclist. The vehicle hits (hits) the cyclist, or the cyclist hits the vehicle.

7. Hitting the car. This includes hitting a moving vehicle and hitting a wild or domestic animal of this type.

8. The fall of the passenger. An accident is caused by a passenger falling from a moving vehicle or by abrupt braking inside the vehicle or changing the trajectory.

9. Other (remaining) traffic accidents. This type of traffic accident includes injuries caused by a tram derailing a vehicle or pedestrian, accidents caused by a load falling from a truck, getting under a car wheel, rocks or other solid objects, and so on enters.

Considering the accident that occurred on the roads under their control (mainly due to the lack of road conditions), the road companies record it in the form of traffic accident -1 or in a separate book in the form of the table below (Table 3.7). An action plan to improve road conditions will be developed by studying the monthly, quarterly, and annual traffic indicators. For a full accounting of traffic accidents in road facilities, the card form (Table 3.8) is recommended in MKN 15-2007 "Rules for accounting and analysis of road accidents", which can be paid in writing or by computer. The advantage of these cards is that all data is entered into a personal computer, setting one or another indicator. The card consists of the following sections: general information, road history, and longitudinal sections, other information about the road, type of road surface, condition of the carriageway, weather information, traffic flow condition, road lighting, description of the place of residence, where the accident occurred a description of the location, the condition of the road and how well it is equipped, the conclusion and the measures to be taken to improve the condition of the road.

The role of computers in the collection and analysis of traffic jams is very large. Now in all developed countries, the use of computers in collecting and analyzing traffic jams is widespread.

A well-designed database allows you to use the available data automatically, specifying the analysis criteria. In the Republic of Uzbekistan, the procedure for the formation, recording, and use of the main indicators of the State of road safety is regulated by a decree of the Cabinet of Ministers of the Republic of Uzbekistan [2]. Previous studies have proven that GIS technologies increase productivity, perform various analyzes and generate graphs in a short time [3-7]. Table 2 shows a tabular form for accounting for road accidents. 
International organizations recommend using the number of deaths per 100,000 of the country's population and the number of deaths per 10,000 vehicles to analyze road accidents. Many studies have examined the relationship between road traffic injuries and other socio-economic indicators. For example, there is a relationship between the overall death rate (deaths per 100,000 populations) and GDP per capita.

The World Bank reports looking at data for 88 countries as a gross domestic product (hereinafter referred to as GDP) per capita increases. One of the main conclusions of this study was identifying a sharp increase in the death rate per capita as GDP increases - but only at low levels of GDP per capita, up to a maximum of 6100-8600 US dollars. After reaching this maximum indicator, the per capita mortality rate begins to decline [4].

This study used the online application "Google Fusion Tables". This application allows you to display the location of the accident on a world map and perform certain data analyses.

Many modern studies have shown that road traffic injuries are multi-faceted and not sufficiently studied; it reflects the dynamic development of many conditions and events, both at the economic and social level in all countries.

The level of motorization has a direct impact on road traffic risk indicators. This relationship, which illustrates the influence of the level of motorization of the population on the road traffic risk indicator, has an interesting factor: if the level of motorization with a low GDP per capita in a country affects the growth of the accident risk coefficient, then the level of motorization with a low GDP per capita in a country reduces this factor (according to more than 50 countries in Europe and Asia [5]).

Recording of accidents on highways can be done using the QGIS program, which is used in geographic information systems. At the same time, the analysis of traffic accidents occurs on highways. It serves to reduce the number of traffic accidents that occur on highways. In the complex of highways, the road is of great importance in overcoming the shortcomings that caused the accident. In the QGIS program, the location of the traffic accident is determined (Figure 1).

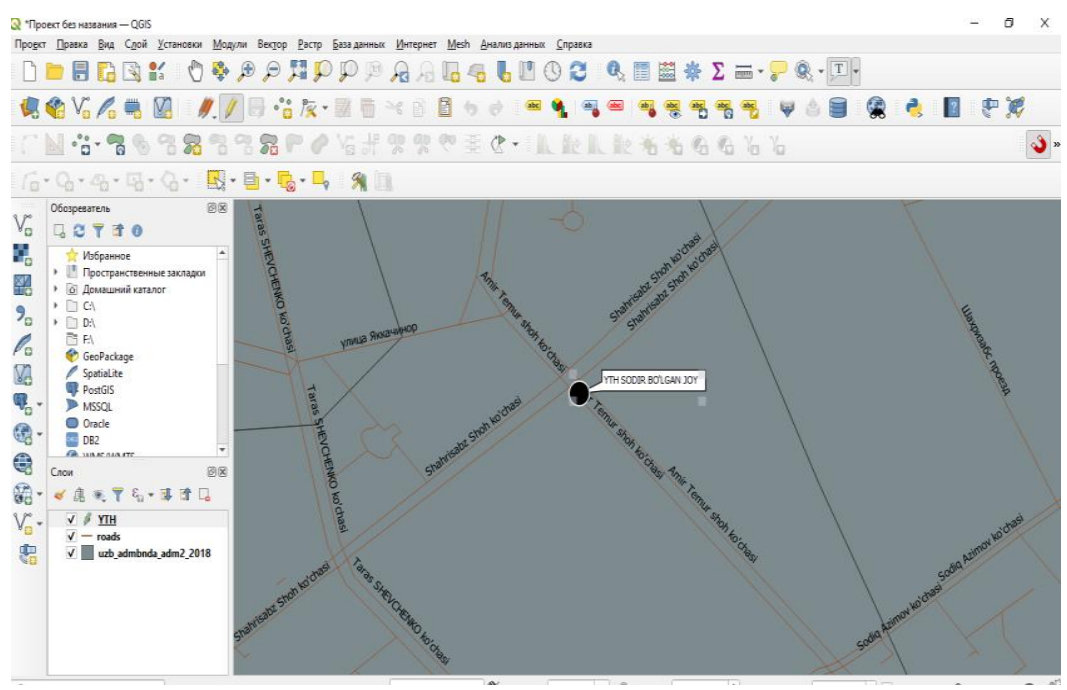




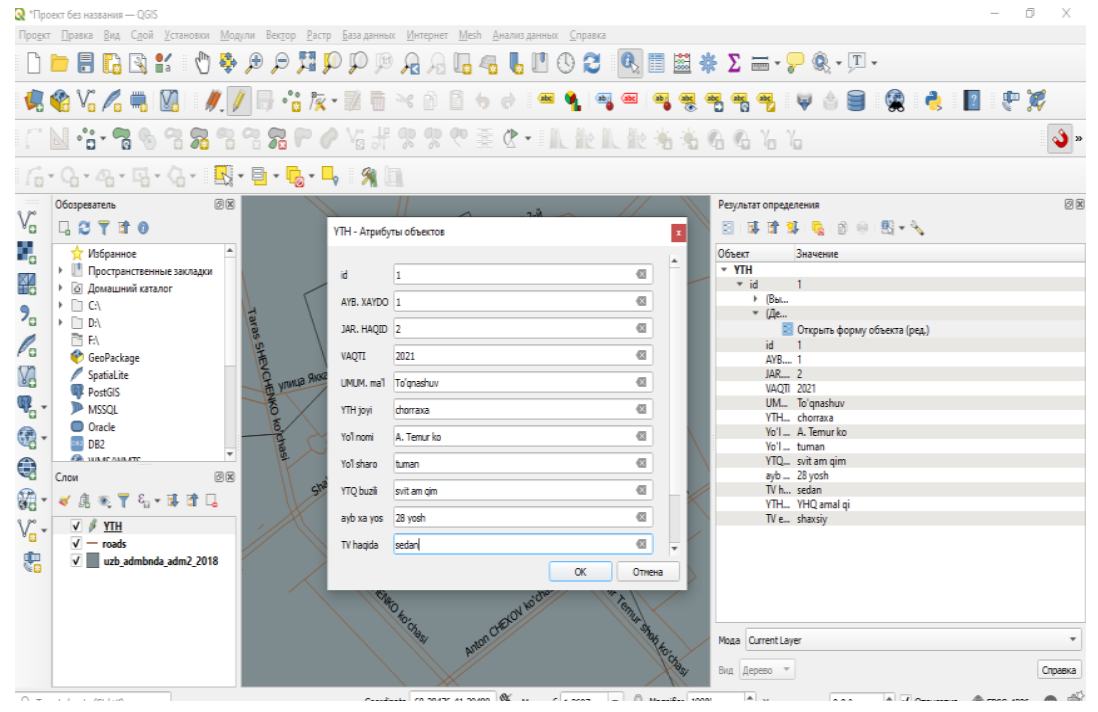

Fig. 1. Using the Qgis program to record traffic accidents that occur on highways

If the results of traffic accidents on highways for a certain period are entered, the data can be shown on the map of the entered area (figure 2).
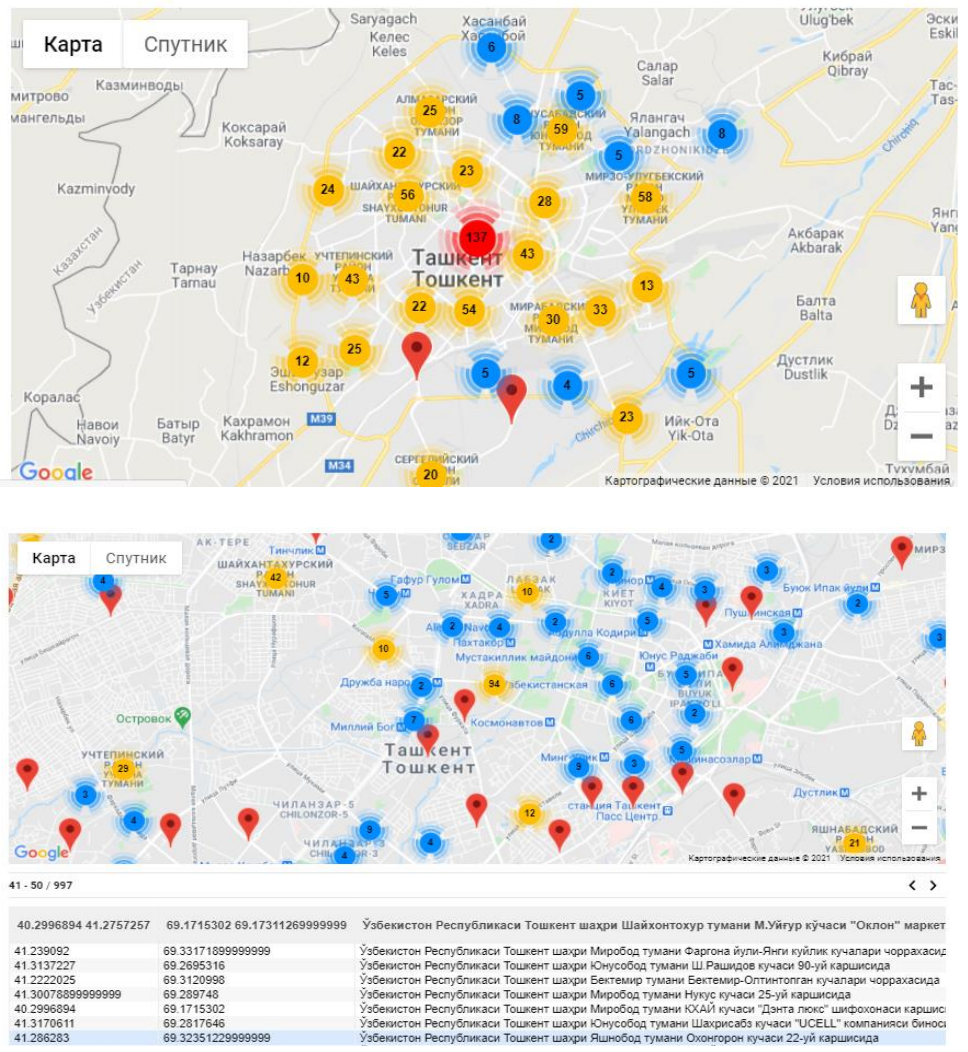

Fig. 2. Mapping of traffic accidents after recording 
There is a big difference between the indicators identified by the researchers on the role of road conditions in the occurrence of traffic accidents and the indicators presented in the state statistics calculations. For example, in the $70 \mathrm{~s}$ and $80 \mathrm{~s}$, researchers showed that 65 $75 \%$ of road conditions played a role in the formation of traffic jams [3], while in former Soviet statistics, it was 7.1-12.1\%. In Uzbekistan, this figure is $0.27-5.22 \%$. Employees of the former All-Union Traffic Safety Research Center reported that $40-45 \%$ of traffic accidents were caused directly or indirectly by large-scale road surveys in 1991 [3], but state statistics accounted for previous levels.

\section{Results and Discussion}

The important factors to road accidents are human, vehicle, and environment. Among them, human is the most significant one. Treat (1980) reports that the human factor contributes to road accidents by $95 \%$. Figure 1 shows the causes of accidents on the road. Causes of accidents remain carelessness, over-speeding, vehicular defect, over- taking and drink driving.

The careless behavior of road users is the main cause of the accident observed from the data analysis. $83 \%$ of the accident happened due to carelessness. Next to this, over speed is another cause of accident occurrence (10\%), followed by the vehicle defect (3\%) and drink driving and overtaking each (2\%). Data analysis from a secondary source (Metropolitan Traffic Police, Singh Durbar) shows that road users' careless driving and walking behaviors are the main causes of accident in this road. These behaviors should be changed to reduce accidents and save the lives of people from accidents. The careless behaviors can be changed by taking extra care outside schools, near buses, and ice cream vans where children might be around.

According to statistics, it is sometimes noted to be YTH due to technical malfunctions of cars. The Bunda is mainly intended for vehicle brakes, Control System, tires, moving aggregate, and displacement. The incorrect operation of the mirror or front view mirror, which describes the faulty heating, cooling system in the car, the unfavorable condition of the driver's seat, the improperly installed rear view, at first glance it seems that Yth is not a reason to come out. But these listed shortcomings worsen the psychophysiological condition of drivers, which ultimately leads directly to the origin of YTH. Unfortunately, in practice, it is considered that in most cases, such factors are caused not by the technical malfunction of the car but by the negligence of the driver.

\section{Conclusions}

There is an increase in an accident immediately after completion of the widening of road and decrease in the successive years. This is because of unfamiliarity among drivers with the increased design speed and unchanged behavior of pedestrians. The rate of accidents is decreasing after the passage of some time due to an awareness program conducted by the project office and media partners focusing safety of school children, bike riders, drivers, and pedestrians. Sallaghari-Thimi and Tinkune-Jadibuti are the sections with a higher number of accidents.

The main contributing factors of road accidents on this road are: carelessness, over speeding, defective vehicle, drink driving, and overtaking. Age is another major cause of accidents; 25 - 34 years of age group is the vulnerable group (41\%). Accidents corresponding afternoon hours (12:00-15:59) are the highest (35\%). Motorbikes' major mode of transportation is involved in accidents (45.5\%). Motorcycles consist of more than 
half number of total vehicles plying in this section and major violation of traffic rule being made by them [8].

Males' involvement in road accidents is higher than females corresponds to the higher males involved- ment in outdoor works and their more aggressive nature. Following recommendations are made to ensure traffic safety along the Kathmandu-Bhaktapur road. 1. Stringent enforcement of the provisions of the VTMA for traffic rule violators. Continue and promote drunken driving testing drive. 2. Priorities for behavioral changes by immediate actions such as road campaigns and visual demonstrations.

From the observation of the highway, the following is achieved: drawing up a passport of the road; drawing up a scheme for the placement of road signs and unloading of road signs; drawing up a scheme for the complete equipping of the highway; determining the possibility of carrying large carriages or large cargoes from a heavy load; determining the type of repair and maintenance work; ; to determine the compliance of the road or its elements with the requirements of architecture; to determine the mode of transport flow in different sections of the road; to assess the quality of transport-exploitation of the road; to assess the consumption of side oil on the road or its parts, etc. the G.

Examination program, determining the size and duration of the work in it; set the order of the examination and drawing up a calendar schedule; drawing up the composition of the examination group (expedition); preparation of the necessary measuring instruments, equipment for the examination and bringing it to the working State; preparation of necessary journals, forms for field work; study of cartographic, Metrological materials, as well as

Conduct awareness and training programs for drivers to raise the level of understanding and skills. 3. Installation of traffic signs in each section and grade-separated intersections at an intersection.

In summary, the QGIS program is efficient, time-saving, and easy to analyze when recording, collecting, and analyzing road traffic accidents.

\section{References}

1. J.I. Sodikov. Road inventory data representation via QGIS in Tashkent region Highways: safety, environmental problems, economics (Russian-German OPMT)/ed. IN AND. Lukanin, K.Kh. Lenz. M.: Logos, 2002 - 624 pages.

2. V.V. Chvanov. Comparative analysis of international statistics on road traffic accidents. Collection of scientific works of MADI (TU) "Design of highways". M .: $2000-111-120$ p.

3. V.F. Babkov Road conditions and traffic safety: Textbook for universities. M .: "Transport", 1993-271 p.

4. Instructions for traffic safety on highways. MSHN 25-2005 State Joint Stock Company "Uzavtoyul". Tashkent, 2007 - 312 p.

5. P.I. Pospelov, T.V. Samodurova, A.G. Malofeev and others. Fundamentals of computer-aided design of highways (based on the CREDO software complex), study guide M .: 2007 - 216 pages.

6. Ryabchinsky A.I. Regulation of active and passive safety of vehicles: textbook for students of higher education. educational institutions / A.I. Ryabchinsky, B.V. Kisulenko et al. M. ed. Center "Academy", 2006 - 432 pp.

7. B.I. Bozorov Environmental safety of vehicles. Toshkent: TADI, 2005 - 104 pages.

8. J.I. Sodikov. Visualization of road accidents using Google Fusion Tables/

9. Sadikov J.I., Silyanov V. V. Inventory of traffic lights on the streets of Tashkent using GIS technology", Transport Systems of Siberia. The development of the transport system as a catalyst for the growth of the state economy. International scientific and 
practical conference (Krasnoyarsk, April 7-8, 2016): sat. nauch. tr.: at 2 h. Ch. $1 /$ under the general editorship of V. V. Minin. - Krasnoyarsk: Sib. Feder. Univ., 2016. pp. 470-474.

10. O. D. Samarin, Temperature in linear elements of enclosing structures, Mag. Civ. Eng., 70, № 2, pp. 3-10, (2017), doi: 10.5862/MCE.70.1.

11. V. A. Rybakov, I. A. Ananeva, A. O. Rodicheva, and O. T. Ogidan, "Stress-strain state of composite reinforced concrete slab elements under fire activity, Mag. Civ. Eng., 74, № 6, pp. 161-174, (2017), doi: 10.18720/MCE.74.13.

12. E. Nedviga, N. Beresneva, M. Gravit, and A. Blagodatskaya, "Fire Resistance of Prefabricated Monolithic Reinforced Concrete Slabs of Marko' Technology, Adv. Intell. Syst. Comput., 692, pp. 739-749, (2018), doi: 10.1007/978-3-319-70987-1_78.

13. A. V. Bushmanova, D. K. Kharchenko, K. S. Semenov, Y. G. Barabanshchikov, V. K. Korovina, and A. V. Dernakova, Thermal cracking resistance in massive steelreinforced concrete structures, Mag. Civ. Eng., 79, № 3, pp. 45-53, (2018) doi: 10.18720/MCE.79.5.

14. J. J. del Coz-Díaz, J. E. Martínez-Martínez, M. Alonso-Martínez, and F. P. Álvarez Rabanal, Comparative study of LightWeight and Normal Concrete composite slabs behaviour under fire conditions, Eng. Struct., 207, (2020), doi:1016/j.engstruct.2020.110196. 